\title{
A New Spot-Size Converter Concept Using Fiber-Matched Antiresonant Reflecting Optical Waveguides
}

\author{
Marko Galarza, Kurt De Mesel, Steven Verstuyft, Cándido Aramburu, Manuel López-Amo, Senior Member, IEEE,
} Ingrid Moerman, Member, IEEE, Peter Van Daele, Member, IEEE, and Roel Baets, Senior Member, IEEE, Member, OSA

\begin{abstract}
We report on a new concept for InGaAsP-InP 1.55- $\mu \mathrm{m}$ lasers with integrated spot-size converters (SSCs) based on antiresonant reflecting optical waveguides (ARROW). The mode expanders consist of a laterally tapered active region on top of a fiber-matched passive slab waveguide. The large slab mode is laterally confined by an antiresonant configuration of a couple of lateral waveguides defined in the same fabrication process as the active ridge. This feature makes the presented spot-size transformer as simple to fabricate as a standard waveguide, only requiring a planar growth step and a single conventional etch process. The fabricated tapers exhibit a low transformation loss and reduce the coupling loss to standard single-mode fibers from 8 to $4 \mathrm{~dB}$. We also analyze by simulation two variants of the concept proposed in this work, including a taper structure for a buried waveguide, which are expected to show better performance. Simulation results show fiber-coupling efficiencies as low as 2.4 and 1.1 dB for both variants.
\end{abstract}

Index Terms-Optical couplers, optical waveguides, semiconductor device modeling, semiconductor device packaging, semiconductor lasers.

\section{INTRODUCTION}

$\mathbf{T}$ HERE exist two rationales for the use of integrated spot-size converters (SSCs) in the development of low-cost optoelectronic devices. One is the pigtailing or fiber attachment of III-V semiconductor photonic integrated circuits (PIC), mostly active devices containing lasers or semiconductor optical amplifiers; and the other is the integration of such PICs with silica-on-silicon waveguides for the development of hybrid devices. The SSCs provide high coupling efficiencies and large alignment tolerances that enable the use of passive alignment schemes that drastically reduce the packaging cost. The function of an integrated SSC is to enlarge and to reshape the small and asymmetric mode of the III-V semiconductor waveguide component to a large and circular mode that is better adapted to the fiber or silica waveguide. Direct butt-coupling with an untapered device typically introduces $7-10-\mathrm{dB}$ coupling loss. The use of microlenses or tapered/lensed fiber that

Manuscript received July 12, 2002; revised September, 6, 2002. This work was supported in part by Spanish CICYT under Project TIC2001-0877-C02-02 and by a Gobierno de Navarra grant.

M. Galarza, K. De Mesel, S. Verstuyft, I. Moerman, P. Van Daele, and R. Baets are with the Department of Information Technology, Ghent UniversityIMEC, B-9000 Ghent, Belgium (e-mail: mgalarza@intec.rug.ac.be).

C. Aramburu and M. López-Amo are with the Electric and Electronic Department, Public University of Navarre, 31006 Pamplona, Navarra, Spain.

Digital Object Identifier 10.1109/JLT.2003.808614

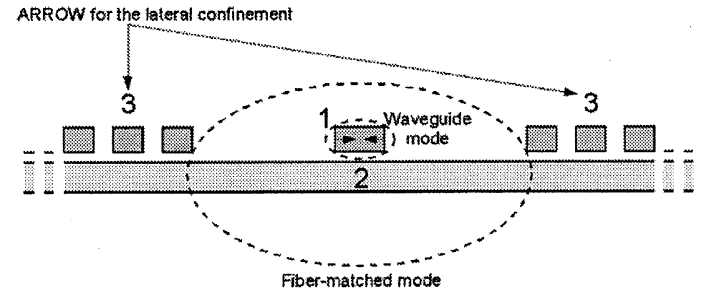

Fig. 1. Schematic (not to scale) of the new SSC concept, using a fiber-matched ARROW waveguide.

reduce the size of the largest mode, improves the coupling loss but at the expense of the alignment tolerance, which remains under the micrometer.

Over the past ten years, much research has focused on the integration of mode size converters with waveguide components in order to improve the coupling efficiency. Most of these approaches involve complex growth and/or processing steps, requiring extensive process development [1]. In this paper, we present a new concept for the fabrication of highly efficient and simple-to-fabricate SSCs by making use of antiresonant reflecting optical waveguides (ARROW) waveguides. We experimentally demonstrate the concept for a $1.55-\mu \mathrm{m}$ InGaAsP-InP laser and analyze and propose, by simulation, two interesting variants of the structure.

\section{CONCEPT}

A schematic of the SSC concept presented in this work is shown in Fig. 1. The device consists of a standard waveguide that is laterally tapered along its propagation direction (region 1 in Fig. 1) and placed on top of a fiber-matched (or silica waveguide-matched) slab waveguide (region 2 in Fig. 1). Two sets of additional ridges are defined on both sides of the central standard waveguide in the same processing step (region 3 in Fig. 1) that creates the tapered ridge one. The adiabatic lateral tapering causes the spreading of the small mode into the broader slab waveguide, increasing its size both laterally and vertically. The lateral guiding of the fiber-matched mode is provided by the two sets of lateral waveguides by means of an antiresonance effect, so this structure can be considered as an ARROW waveguide [2], [3]. As the lateral and central tapered waveguide can be defined in the same processing step, this taper is the simplest two-dimensional (2-D) SSC demonstrated so far. 


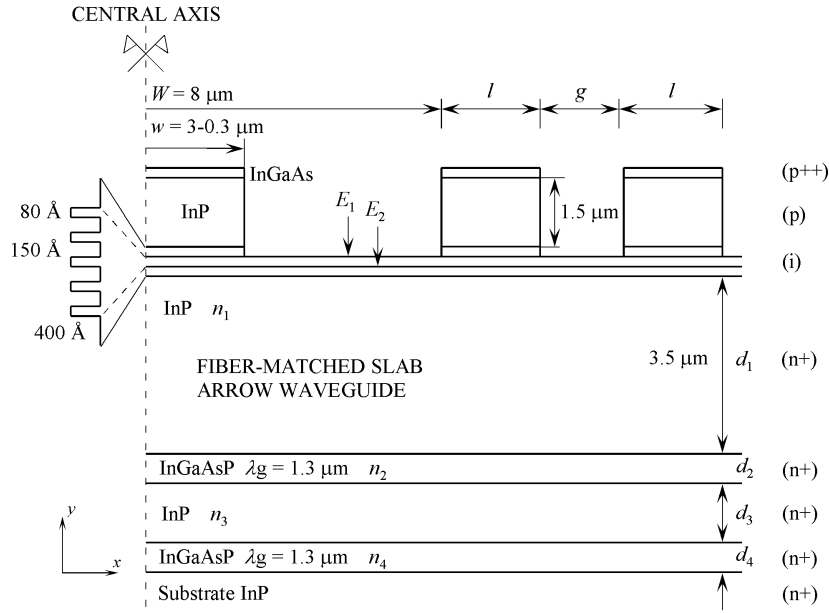

Fig. 2. Schematic drawing of the adiabatic mode-expanded laser showing the tapered upper active rib, the underlying fiber-matched ARROW slab waveguide, and the lateral confinement rib waveguides.

\section{DeVICE DESIGN AND THEORY}

The transverse structure of the proposed mode-expanded laser is shown in Fig. 2. The device consists of an active multiquantum well rib waveguide that is laterally tapered from 3 to $0.3 \mu \mathrm{m}$, and that contains five $1 \%$ compressively strained 80 - $\AA$-thick $\operatorname{In}_{0.78} \mathrm{Ga}_{0.22} \mathrm{As}_{0.79} \mathrm{P}_{0.21}$ wells for emission at $1.55 \mu \mathrm{m}$, and four lattice-matched $150-\AA$-thick $\mathrm{In}_{0.75} \mathrm{Ga}_{0.25} \mathrm{As}_{0.54} \mathrm{P}_{0.46}$ barriers, surrounded by 400 - $\AA$-thick undoped confinement layers with the same composition as the barriers.

The underlying passive slab waveguide is realized by a standard vertical ARROW waveguide [4]. A quaternary compound with $\lambda_{g}=1.3 \mu \mathrm{m}$ for the two high-refractive index ARROW layers was chosen. The thickness of the ARROW core was limited to $3.5 \mu \mathrm{m}$ to avoid the loss of the lateral confinement effect when the slab core becomes too thick. The thickness of the corresponding cladding layers are: $d_{2,4}=0.32 \mu \mathrm{m}$ and $d_{3}=1.86 \mu \mathrm{m}$ (TE polarization) [4]. The total epitaxial layer thickness is not attractive form a growth point of view, but we already demonstrated in previous work that they could be considerably reduced without altering the performance of the device [5].

A couple of appropriately designed rib waveguides placed on each side of the tapered central waveguide provides the lateral confinement of the guided mode. The behavior cannot be explained with the standard vertical ARROW formulas because of the guided modes that appear in the lateral ribs. Considering 2-D coupled-mode theory, we can treat the new mode intuitively as an antisymmetrical combination of the coupled modes of the upper lateral rib waveguides and the mode of the underlying InP slab ARROW core [6]. Thus, the shape of the output mode depends strongly on the width of the lateral ribs. Fig. 3 shows the confinement of the fiber-matched leaky mode in the quantum wells (QWs) of the lateral ribs for the structure of Fig. 2 as a function of the lateral rib width $l$, while keeping the gap $g$ constant and equal to $1 \mu \mathrm{m}$. This confinement should be minimized in order to reduce absorption loss in the unpumped lateral ribs.
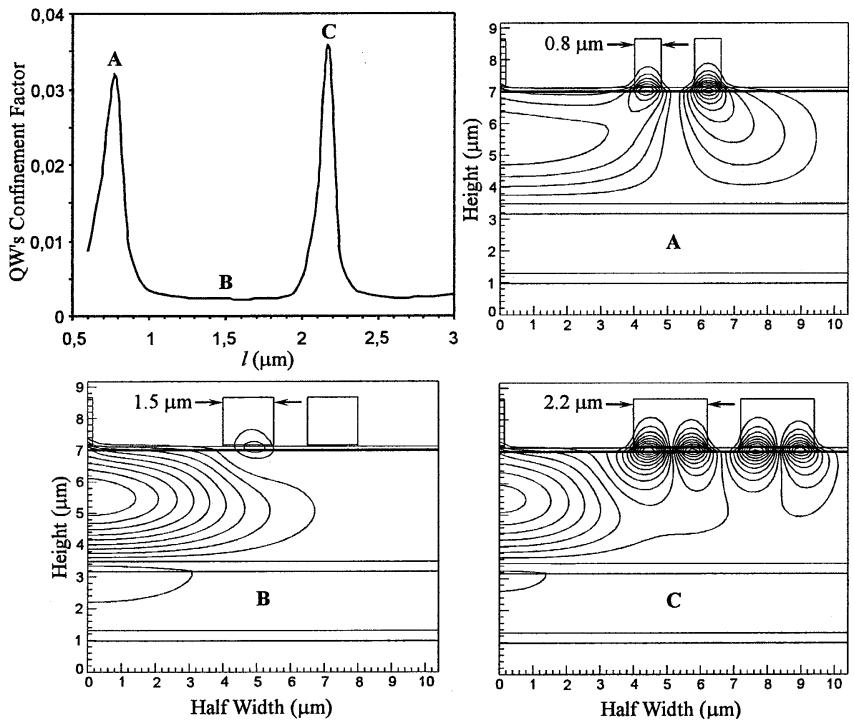

Fig. 3. Confinement of the output mode in the QWs of the active layer of a shallow-etched device as a function of the widths of the lateral ribs $l$, maintaining the gap $g$ between ribs constant. The field distributions of the modes at the points indicated in the graph are also shown.

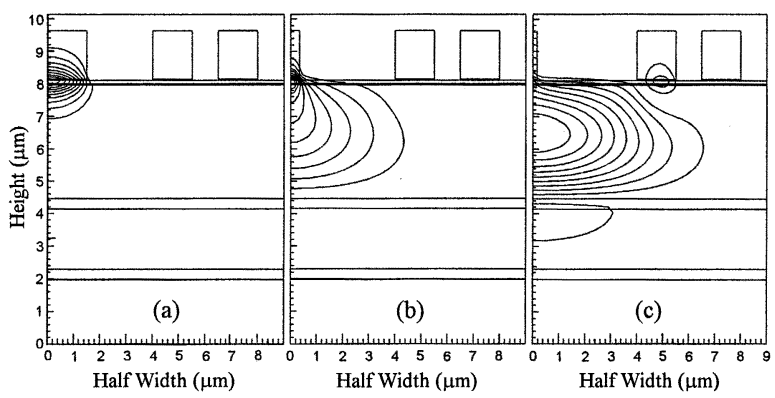

Fig. 4. Fundamental TE mode evolution along the taper structure of Fig. 2 for (a) $w=3 \mu \mathrm{m}$, (b) $w=0.9 \mu \mathrm{m}$, and (c) $w=0.3 \mu \mathrm{m}$. A shallow etch is simulated.

The field profiles for different widths $l$ are also shown for illustration. A flat and broad minimum confinement is found around $l=1.5 \mu \mathrm{m}$, where the output mode is fit for coupling to fiber. The broad minimum implies that the device is tolerant to fabrication errors. Two designs, with a different etch depth for the laser rib, are considered (shown as $E_{1}$ and $E_{2}$ in Fig. 2) and the optimum width $l$ for the deep-etched device is also calculated and found to be $1.6 \mu \mathrm{m}$. In both etch options, the optimum gap $g$ between lateral ribs is less than $1 \mu \mathrm{m}$, but has to be increased to $1 \mu \mathrm{m}$ because of fabrication limitations. Fortunately, this does not change the modal shape significantly.

The evolution of the optical field as a function of the upper central rib is shown in Fig. 4. As the active waveguide is tapered by reducing the ridge width from 3 to $0.3 \mu \mathrm{m}$, the mode couples adiabatically from the upper guide to the underlying ARROW waveguide (see Fig. 4). The design of the lateral tapering is based on the adiabaticity of the mode transformation, and has been evaluated by means of a commercial three-dimensional (3-D) eigenmode expansion algorithm based on a resonance method [7]. Low taper angles are needed for the range where the modal transformation is produced, in order to avoid 


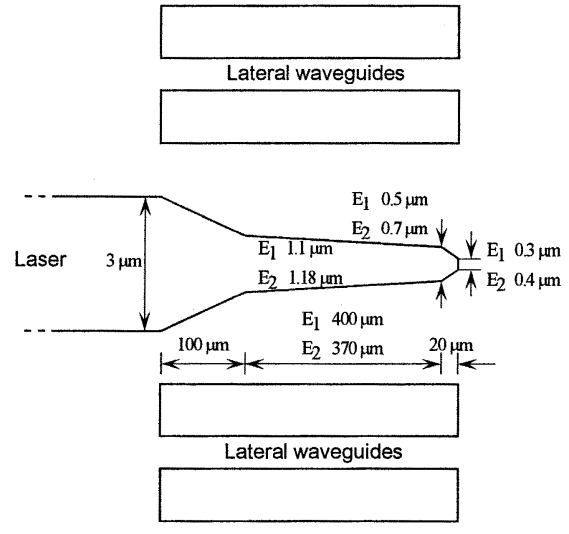

Fig. 5. The optimum taper shape is approximated by a piecewise linear device consisting of three linear sections. Tapers for both shallow-etched $\left(E_{1}\right)$ and deep-etched $\left(E_{2}\right)$ laser are designed.

radiation losses, while higher angles are allowed in the other regions. The adiabatic shape designs for both etching options have been approximated by a piece-wise linear device consisting of three linear sections and are sketched in Fig. 5. A minimum narrowing of $100 \mathrm{~nm}$ per $77-\mu \mathrm{m}$ propagation length yields a safe design from the fabrication point of view. Both designs exhibit a calculated transformation loss of $1 \mathrm{~dB}$.

When the mode is expanded in the shallow etched device, it will be slightly confined in unpumped active quaternary layers [see Fig. 4(c)]. This may introduce some absorption losses in the shallow-etched lasers that are not present in the deep-etched designs.

\section{Device Processing}

The epitaxial layers were grown by metal-organic chemical vapor deposition (MOCVD). By plasma etching, we defined the $\mathrm{SiO}_{x}$ pattern with conventional photoresist as a mask. The central and lateral ribs were etched by reactive ion etching (RIE) to a depth $100 \mathrm{~nm}$ above the active layer. Next, a selective wet etch of the remaining InP down to the confining layer of the active layer was carried out. This etch was done using $\mathrm{H}_{3} \mathrm{PO}_{4}$ : $\mathrm{HCl}$ (7:3). Finally, an extra 40 (shallow etch) or $140 \mathrm{~nm}$ (deep etch) of RIE etching into the active layer was performed. Next, polyimide was spin coated on the sample to form an insulation layer. The thickness of the polyimide is less on top of the ridges compared to the rest of the sample. Therefore, by a controlled plasma etch, the dielectric was removed on the ridge while dielectric coverage remained elsewhere resulting in good electric isolation [8]. Scanning electron microscope (SEM) pictures of the taper (Fig. 6) revealed that the metal contact over this upper ridge was good and that the thin ribs were fabricated reproducibly. Next, using a negative photoresist and a metal liftoff process, the metallization pattern was defined. Finally, the thinning of the substrate was done and the back contact was deposited. Arrays of devices, each with $520 \mu \mathrm{m}$ long straight active sections, were mounted on electrically cooled copper heatsinks with silver epoxy and were tested without any coatings. The entire device is electrically pumped because the metallization also covers the entire length of the SSC. This can have a negative impact on the threshold current and efficiency, but on the other hand, it avoids the risk of high absorption losses

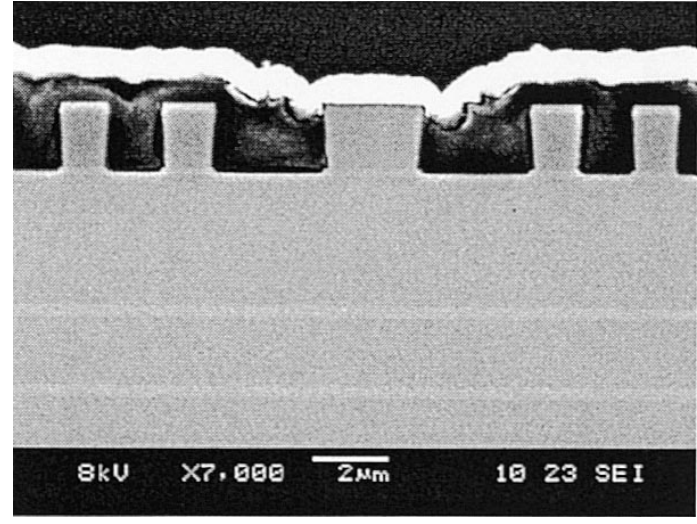

Fig. 6. SEM image of the laser cross section, showing the electric contact over the central tapered rib, the good definition of the lateral ribs, and the high index ARROW cladding layers.

TABLE I

MEASUREMENT AND SimUlation Results on the INTEGRATEd Mode EXPANDED LASERS CONSIDERED IN THIS WORK

\begin{tabular}{lcccccc}
\hline Laser & $\begin{array}{c}I_{t h} \\
(\mathrm{~mA})\end{array}$ & $\begin{array}{c}\eta_{d} \\
(\mathrm{~W} / \mathrm{A})\end{array}$ & $\begin{array}{c}L \\
(\mu \mathrm{m})\end{array}$ & $\begin{array}{c}F C \\
(\mathrm{~dB})\end{array}$ & $\begin{array}{c}F F \\
(\mathrm{Deg} .)\end{array}$ & $\begin{array}{c}\text { Tol. } \\
(\mu \mathrm{m})\end{array}$ \\
\hline Reference, $E_{1}$ & 35 & 0.12 & 0 & -8 & $31.5 \times 42.8$ & $\pm 1.6 \times \pm 2$ \\
Reference, $E_{2}$ & 28 & 0.12 & 0 & -8.3 & $30.7 \times 40.6$ & $\pm 1.5 \times \pm 2$ \\
\hline Tapered, $E_{1}$ & 55 & 0.1 & 520 & -4 & $13.8 \times 30.8$ & $\pm 2.3 \times \pm 2.4$ \\
Tapered, $E_{2}$ & 48 & 0.12 & 490 & -4.3 & $13 \times 32.7$ & $\pm 2.2 \times \pm 2.3$ \\
\hline Variant 1 (Fig. 9) & - & - & 490 & -2.4 & $7.2 \times 14$ & $\pm 2.3 \times \pm 1.9$ \\
Variant 2 (Fig. 10) & - & - & 270 & -1.1 & $7.2 \times 9$ & $\pm 2.9 \times \pm 2.3$ \\
\hline
\end{tabular}

in the passive section of the taper. Untapered lasers $750 \mu \mathrm{m}$ long and $3 \mu \mathrm{m}$ wide and fabricated from the same wafer as the tapered lasers were also prepared as a reference. Both reference and tapered lasers have a similar total active area.

\section{RESULTS AND DISCUSSION}

All the measured parameters are shown in Table I. The devices were operated in continuous-wave $(\mathrm{CW})$ operation. The room temperature light intensity versus current $(L-I)$ curves of mode-expanded and untapered reference lasers are shown in Fig. 7 for both etch depths. The deep-etched reference devices, with a threshold current of $28 \mathrm{~mA}$, present a better laser performance than the shallow etched ones ( $35 \mathrm{~mA})$. Fairly uniform threshold current distributions were found along the laser array.

Regarding the mode-expanded lasers, an increase of about $20 \mathrm{~mA}$ in the threshold current with respect to the reference lasers is found. This can be attributed to the mode transformation loss within the taper, to the losses in the n-doped-thick InP ARROW core layer, and to the nonuniform pumping of the tapered active rib as a function of its width. The fact that the power of the tapered device is measured at the expanded mode facet, which has a lower reflection coefficient than the reference laser facet, influences positively in the external efficiency of the lasers. Nevertheless, the higher absorption loss in the unpumped QWs of the lateral ARROW rib waveguides for the shallow- 


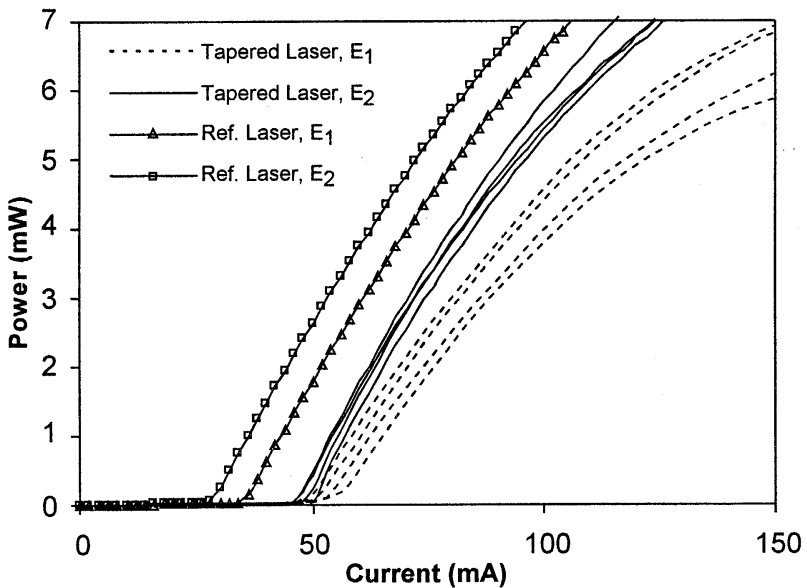

Fig. 7. $L-I$ characteristics of several shallow-etched $\left(E_{1}\right)$ and deep-etched $\left(E_{2}\right)$ mode-expanded and untapered reference lasers.

etched devices [see Fig. 4(c)] causes a decrease of the external efficiency of the laser from $0.12 \mathrm{~W} / \mathrm{A}$, for the reference lasers, to $0.1 \mathrm{~W} / \mathrm{A}$. This fact is not observed in the deep-etched devices that maintain the same external efficiency as the reference lasers.

Far-field emission patterns for both tapered and reference lasers were measured by using a rotating stage and a pinhole detector (see Fig. 8). The theoretical curves obtained from the Fourier transform of the theoretical near fields are also overlaid in Fig. 8, for comparison. The measured full-width at half-maximum (FWHM) values are shown in Table I. A considerably lateral divergence improvement is achieved in the lateral direction for both groups of lasers, reaching a minimum of $13^{\circ}$ for the deep-etched lasers. On the other hand, due to the not so thick ARROW slab core $(3.5 \mu \mathrm{m})$, the achieved vertical field divergence is still slightly higher than $30^{\circ}$, improving it by $10^{\circ}$ with respect to the reference laser. A further improvement in the vertical far-field pattern could be obtained by thickening the ARROW core layer and defining more than two ribs on each side of the output ARROW waveguide in order to provide a good lateral confinement for thick cores.

A coupling efficiency measurement was performed between the expanded mode laser and a standard cleaved single-mode fiber (SMF) with a spot size of $10 \mu \mathrm{m}$ at $1.55 \mu \mathrm{m}$. Maximum fiber coupling efficiencies of around $-4 \mathrm{~dB}$ are obtained for both etch depths, including the Fresnel losses occurring at the air-glass interface. The improvement in the fiber coupling with respect to the untapered devices amounts up to $4 \mathrm{~dB}$ (see Table I). As the alignment tolerance with respect to the fiber depends on the large fiber mode, only slight improvements in the lateral direction are observed with respect to the reference untapered lasers. A maximum 1-dB alignment tolerance of \pm 2.3 (lateral) $\times \pm 2.4$ (vertical) is obtained for the shallow-etched devices. The fact that the vertical alignment tolerance is higher than the lateral value is due to the fact that the fiber facet was somewhat moved away from the taper output facet. These measurement restrictions were considered to avoid the contact of the fiber end with the laser output facet during the sweep of the transverse position of the fiber.

All the results are in good agreement with theoretical calculations.

\section{VARIANT 1: WEAKLY GUIDING FIBER-ADAPTED WAVEGUIDE}

The SSC concept proposed in this work has been demonstrated experimentally as discussed in the last section. Nevertheless, the waveguide structure of Fig. 2 has a limitation on the vertical direction because the lateral ribs lose their confinement effect when the slab core becomes too thick. The structure of Fig. 9 is similar to that of Fig. 2, but it makes use of a thin weakguiding core of only $80 \mathrm{~nm}$ and buried in InP beneath the active layer. The mode at the active rib and the mode at the end of the tapering are also shown. The MQW gain in the straight section of the laser remains unalterable (see Figs. 4(a) and 9(a) for comparison). The fiber-matched mode presents a quite asymmetrical field distribution in the vertical direction, but the fact that its maximum is closer to the upper lateral couple of ribs strengthens their influence. Moreover, from a coupling and far-field point of view the typical exponential decay of weak-guiding waveguides induces an improvement of the coupling efficiency and FWHM of the output beam, in spite of its asymmetrical shape.

Simulation results on the propagation of this new structure are shown in Table I. Only the design for the deep etching through the QWs has been considered (see Fig. 2) because of its better lasing performance. The same tapering sketched in Fig. 5 has been simulated, because it also provides an adiabatic mode transformation for this new structure. We observe that the beam divergence angles and the coupling efficiency are improved to $7.2^{\circ} \times 14^{\circ}$, and $-2.4 \mathrm{~dB}$, respectively. Due to the vertically asymmetric modal shape, the vertical alignment tolerance is slightly reduced to $\pm 1.9 \mu \mathrm{m}$, while the lateral value is maintained.

\section{VARIANT 2: BURIED LASER}

The mode transformation concept proposed in the present work can also be integrated in a second variant, being a buried laser structure as shown in Fig. 10. A buried 194-nm-thick MQW active layer having an equivalent refractive index of 3.45 is tapered from 1 to $0.2 \mu \mathrm{m}$ following the tapering shown in the inset of Fig. 10(a). The slab waveguide core of quaternary compound with $\lambda_{g}=1.1 \mu \mathrm{m} \mathrm{Q}(1.1)$ is buried $0.2 \mu \mathrm{m}$ beneath the active core and is $70 \mathrm{~nm}$ thick. The optimum lateral confinement ARROW ribs are $0.9 \mu \mathrm{m}$ wide and are separated by $2 \mu \mathrm{m}$. All this is embedded under a 5- $\mu \mathrm{m}$-thick top layer of InP.

The fact that the tapered active central core is totally embedded in InP makes the mode transformation efficiency much higher for buried than for rib structures. This is the reason why the length of the buried SSC $(270 \mu \mathrm{m})$ is much shorter than the rib SSC $(490 \mu \mathrm{m})$.

Simulation results on the SSC propagation are shown in Table I. They show fiber butt-coupling losses as low as $1.1 \mathrm{~dB}$, beam divergence angles of $7.2^{\circ} \times 9^{\circ}$, and alignment tolerances of $\pm 2.9 \times \pm 2.3 \mu \mathrm{m}$, compared to the 8.5 - $\mathrm{dB}$ coupling loss and $29^{\circ} \times 32^{\circ}$ divergence angles of the untapered devices.

\section{CONCLUSION}

In this paper, we have presented a new concept for the fabrication of highly efficient and simple-to-fabricate SSCs that make 

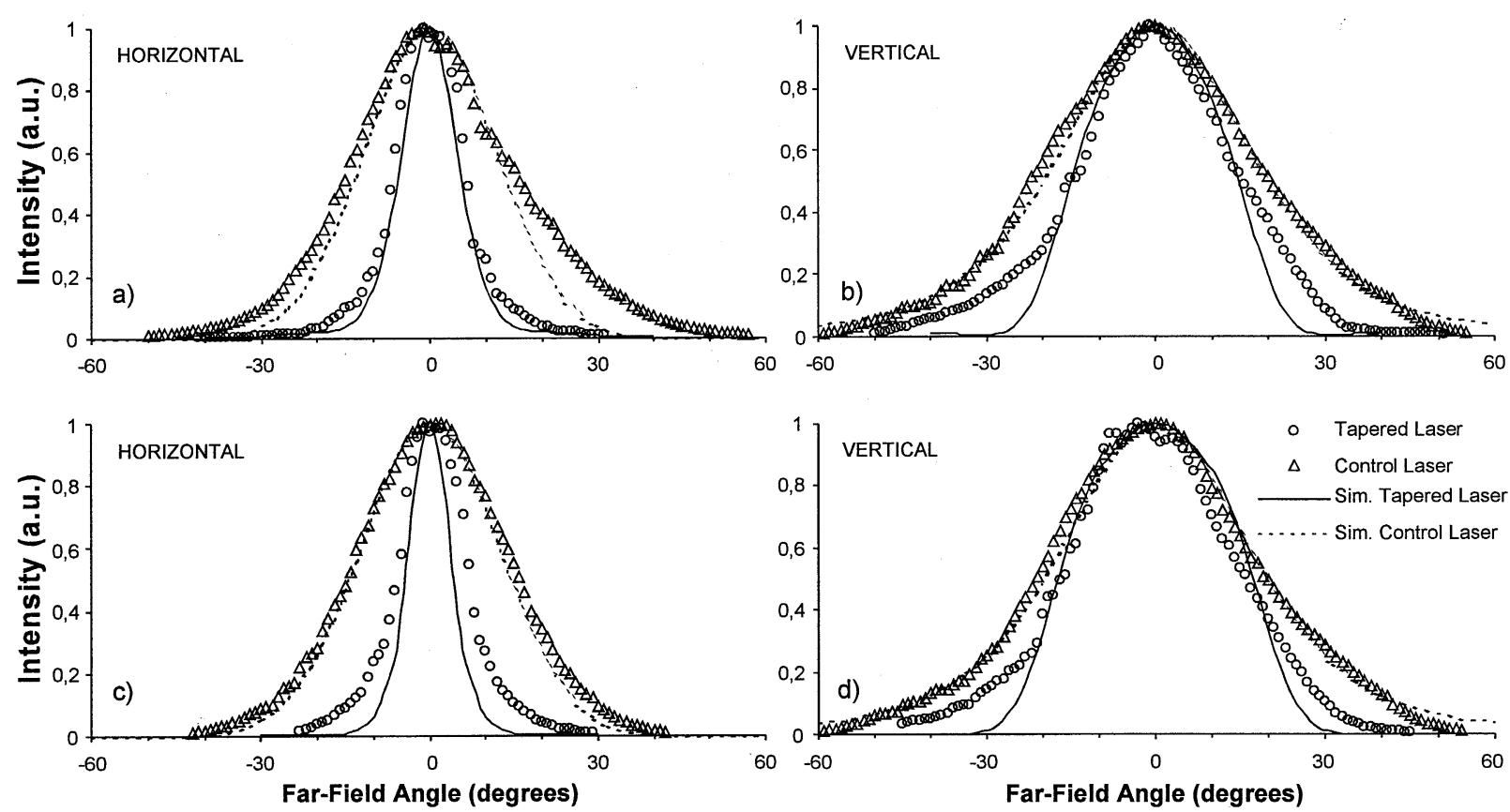

Fig. 8. Far-field emission patterns of tapered and untapered lasers. Shallow-etched lasers (a) lateral and (b) vertical. Deep-etched lasers (c) lateral, and (d) vertical.

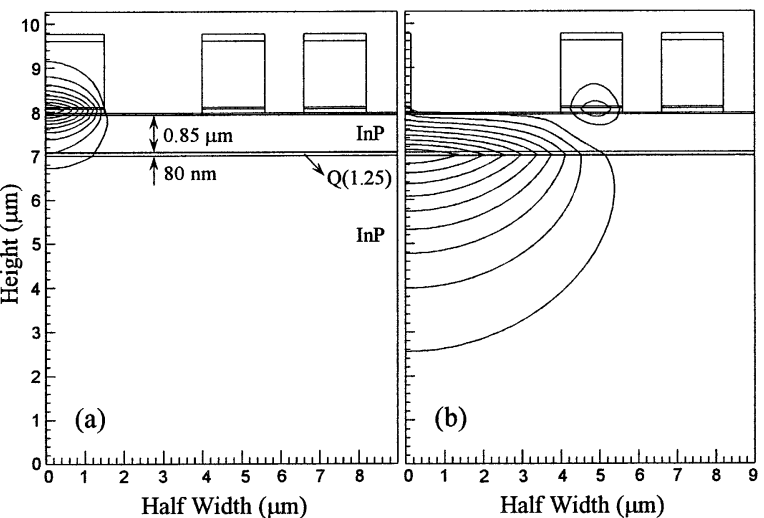

Fig. 9. Schematic structure of the SSC using a weakly guiding underlying slab waveguide. The active layer and upper rib waveguides are identical to that of Fig. 2.

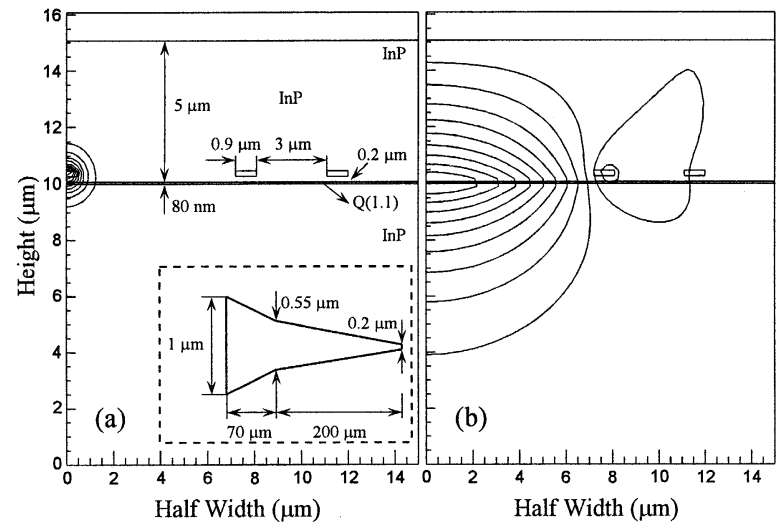

Fig. 10. Schematic structure of a buried variant of the proposed SSC concept. Higher and more efficient mode transformation efficiencies can be achieved in buried structures.

use of the ARROW-concept for both the lateral and vertical optical confinement. InGaAsP-InP rib lasers with monolithically integrated SSCs have been demonstrated. The taper transforms the small active laser mode into the fiber-adapted mode of the underlying slab ARROW waveguide. The lateral confinement of the slab mode is achieved by means of an antiresonant effect provided by two sets of lateral rib waveguides defined in the same etch process as the central active ridge. Therefore, only a single planar growth step and a single conventional etching process are required, leading to the simplest SSC concept ever reported. Threshold currents of around $50 \mathrm{~mA}$ were measured, which have to be compared with $30 \mathrm{~mA}$ for untapered lasers. The far-field FWHM divergence angles were significantly reduced and a 4-dB improvement of the coupling efficiency to SMF was observed.

Two variants of the demonstrated concept have also been analyzed by simulation and are expected to show a better performance. In a first variant, a weak-guiding slab waveguide substitutes the thicker slab core of the first structure. In a second variant, the SSC concept of this work is integrated in a buried laser structure. Simulation results show considerable improvements in the far-field divergence angles and fiber-coupling efficiencies.

\section{REFERENCES}

[1] I. Moerman, P. Van Daele, and P. M. Demeester, "A review on fabrication technologies for the monolithic integration of tapers with III-V semiconductor devices," IEEE J. Select. Topics Quantum Electron., vol. 3, pp. 1308-1320, Dec. 1997.

[2] I. V. Golster, L. J. Mawst, and D. Botez, "Single-cladding antiresonant reflecting optical waveguide-type diode laser," Opt. Lett., vol. 20, pp. 2219-2221, Nov. 1995.

[3] M. Galarza, K. De Mesel, R. Baets, and M. Lopez-Amo, "Modeling of InGaAsP-InP $1.55 \mu \mathrm{m}$ lasers with integrated mode expanders using fiber-matched antiresonant reflecting optical waveguides," in Proc. Eur. Conf. Integrated Optics (ECIO '01), Paderborn, Germany, pp. 421-424.

[4] J. M. Kubica, "Numerical analysis of InP/InGaAsP ARROW waveguides using transfer matrix approach," J. Lightwave Technol., vol. 10, pp. 767-771, June 1992. 
[5] M. Galarza, K. De Mesel, D. Fuentes, R. Baets, and M. Lopez-Amo, "Modeling of InGaAsP-InP $1.55 \mu \mathrm{m}$ lasers with integrated mode expanders using fiber-matched leaky waveguides," Appl. Phys. B. Lasers Opt., vol. B73, pp. 585-588, Oct. 2001.

[6] G. R. Hadley, "Two-dimensional coupled-mode theory for modeling leaky-mode arrays," Opt. Lett., vol. 15, pp. 27-29, Jan. 1990.

[7] A. S. Sudbo, "Numerically stable formulation of the transverse resonance method for mode-field calculations in dielectric waveguides," IEEE Photon. Technol. Lett., vol. 5, pp. 342-344, Mar. 1993.

[8] V. Vusirikala, S. S. Saini, R. E. Bartolo, S. Agarwala, R. D. Whaley, F. G. Johnson, D. R. Stone, and M. Dagenais, "1.55- $\mu \mathrm{m}$ InGaAsP-InP laser arrays with integrated-mode expanders fabricated using a single epitaxial growth," IEEE J. Select. Topics Quantum Electron., vol. 3, pp. 1332-1343, Dec. 1997.

Marko Galarza was born in Urdiain, Spain, in 1973. He received the Ingeniero de Telecomunicación degree from the Public University of Navarra, Pamplona, Spain, in 1997. He is currently pursuing the Ph.D. degree in electrical engineering at the Information Technology Department of the University of Ghent, Belgium.

Until 2000, he has been with the Public University of Navarra, Pamplona, where he worked in the field of integrated optics.
Manuel López-Amo (SM'96) was born in Madrid, Spain, on June 1960. He received the Ingeniero de Telecomunicación degree, and the Ph.D. degree in telecommunications engineering, both from the Universidad Politécnica de Madrid, in 1985 and 1989, respectively.

From 1985 to 1989, he has been a lecturer in optical communications and electronics at the Photonic Technology Department, Universidad Politécnica de Madrid. During 1987, he was a visiting Ph.D. student at the Laboratorium Voor Elektromagnetisme en Acustica of the University of Ghent (Belgium), working on integrated optics. In January 1990, he became an Associate Professor in the Photonic Technology Department, Universidad Politécnica de Madrid. From 1990 to 1995, he was teaching Optical Communications for undergraduated and postgraduated students. In 1992, he was a Visiting Researcher at British Telecom. Research Labs, Ipswich, U.K., working in amplified fiber-optic networks. In March 1996, he obtained a chair as Full Professor at the Universidad Publica de Navarra, Pamplona, Spain, in the Electrical and Electronical Engineering Department, and is now the Head of this department. He has coauthored more than 100 works in journals and conferences all related with fiber-optic networks, fiber-optic components, optical amplifiers, fiber-optic processors, fiberoptics sensors, and integrated optics.

Ingrid Moerman (M'96) was born in Gent, Belgium, in 1965. She received the electrical engineering and Ph.D. degrees from the Ghent University, Gent, Belgium, in 1987 and 1992, respectively.

Since 1987, she has been with the Interuniversity Micro-Electronics Centre (IMEC), Department of Information Technology (INTEC), Ghent University. Her main contributions are the epitaxial growth of III-V optoelectronic components and photonic integrated circuits for telecom applications. In 1997, she became a permanent member of the Research Staff at IMEC, where she coordinates the research on the epitaxial growth of III-V optoelectronic devices. Since 2000, she is a part-time Professor at Ghent University. She has recently expanded her research domain to broad-band communication networks and, more specifically, mobile communications. She is author or coauthor of more than 250 publications in the field of optoelectronic components and technology.

Peter Van Daele (M'91) received the Ph.D. degree in electrical engineering from the University of Ghent, Gent, Belgium, in 1998.

He became a Permanent Member of Staff of the Department of Information Technology, Interuniversity MicroElectronics Centre (IMEC), University of Ghent, where he was responsible for the research on processing of III-V optoelectronic devices. Since 2001, his work has more focused on optical packaging and optical interconnections, with emphasis on coupling to fiber arrays, integration on printed circuit boards, and use of laser processing techniques. In 1993, he also became a part-time Professor. He is author or coauthor of about 200 publications in the field of optoelectronic components and technology.

Roel Baets (M'88-SM'96) received the electrical engineering degree from Ghent University, Gent, Belgium, in 1980, the M.Sc. degree in electrical engineering from Stanford University, in 1981, and the Ph.D. degree from Ghent University in 1984.

Since 1981, he has been with the Department of Information Technology (INTEC), Ghent University. Since 1989, he is a Professor with the engineering faculty of Ghent University. From 1990 to 1994, he has also been a part-time professor at the Technical University of Delft, The Netherlands. He has mainly worked in the field of III-V devices for optoelectronic systems. He has authored 300 publications and conference papers, and has made contributions to the design and fabrication of semiconductor laser diodes, passive guided wave devices, PICs and microoptic components patents. He leads the Optoelectronic Components and Systems group at Ghent University-INTEC (which is an associated lab of IMEC), working on photonic devices for optical communication and optical interconnect.

Dr. Baets is a Member of the Optical Society of America (OSA), IEEE-LEOS, SPIE, and the Flemish Engineers Association. He has been a member of the program committees of OFC, ECOC, IEEE Semiconductor Laser Conference, ESSDERC, CLEO-Europe, and the European Conference on Integrated Optics. From 1999 to 2001, he has been Chairman of the IEEE-LEOS-Benelux chapter.
Cándido Aramburu was born in San Sebastián, Spain, in July 1964. He received the Ingeniero de Telecomunicación degree from the Universidad Politécnica de Madrid, in 1989, and the Ph.D. degree in Telecommunications Engineering from the Public University of Navarre, Pamplona, Spain, in 2001.

From 1992 to 1994, he has been an Associate in the Computer Department, and since 1995, he has been with the Electronics and Photonics Devices in the Electrical and Electronical Engineering Department, Universidad Pública de Navarra. Since 1995, he has worked in the field of integrated optics with emphasis in the InGaAsP-InP passive and active devices. 